\title{
Modelisation des paramètres de fonctionnement d'un cuiseur solaire de type boîte sous les conditions météorologiques du Burkina Faso
}

\author{
J. NEBIE ${ }^{1,2}$, T. DAHO ${ }^{1}$, G.C. TUBREOUMYA ${ }^{1,2}$, S. ZONGO $^{1}$, B. ZEGHMATI ${ }^{2}$, X. CHESNAU $^{2}$, \\ A. O. DISSA ${ }^{1}$, S. Z. KAM ${ }^{1}$, A. BERE ${ }^{1}$. \\ ${ }^{1}$ Laboratoire de Physique et de Chimie de l'Environnement (LPCE), Université Joseph KI-ZERBO, 03 BP : 7021 Ouagadougou 03, Burkina Fas \\ ${ }^{2}$ Université de Perpignan Via Domitia : 52, avenue Paul Alduy-66100 Perpignan, France
}

\section{INFOS SUR L'A R T I C L E}

Historique de l'article:

Reçu en format revisé le : 12 décembre 2019

Accepté le : 16 décembre 2019

Mots-Clés:Cuiseur solaire, Type boîte, Simulation,Conditions météorologiques
Reçu le : 31 juillet 2019

\begin{abstract}
RES U M E / A B S T R A C T
Ce travail porte sur la modélisation des paramètres de fonctionnement d'un cuiseur solaire de type boîte, double vitrage, sous les conditions météorologiques du Burkina Faso (Ouagadougou). L'analyse de sensibilité a été faite pour certains paramètres comme l'angle d'inclinaison de la surface réceptrice du cuiseur, la vitesse du vent, l'espace entre les deux vitres, l'épaisseur de l'isolant. Les résultats montrent que le cuiseur solaire de type boîte à surface réceptrice inclinée atteint des niveaux de températures exploitables pour la cuisson. Aussi, ce type de cuiseur donne les meilleures performances sous un angle d'inclinaison de la surface réceptrice voisin de la latitude du lieu. Une épaisseur d'isolant de $6 \mathrm{~cm}$, avec une épaisseur d'air séparant les deux vitres compris entre 0,5 et $1,5 \mathrm{~cm}$ sont adéquats pour une bonne isolation thermique du cuiseur. Enfin, les résultats montrent que la vitesse du vent a une influence négligeable sur ce type de cuiseur.
\end{abstract}

\section{INTRODUCTION}

Les combustibles ligneux représentent environ $90 \%$ de la consommation d'énergie dans la plupart des ménages dans les pays en développement (FAO, 2010). Au Burkina Faso, 85,5\% des ménages utilisent la biomasse (bois énergie, résidus agricoles) pour la cuisson (Malo, 2015). La croissance accélérée de la population et le faible rendement des systèmes classiques de cuisson entrainent une pression sur les formations ligneuses. Ce qui est un vecteur de déforestation et d'accélération de la désertification dans les pays subsahariens. A cela s'ajoute les maladies liées aux fumées dégagées par la combustion de biomasse qui causent plus de 3,5 millions de décès par an dans le monde (Otte, 2013). De plus, le contexte énergétique, climatique et sécuritaire actuel ne laisse pas de choix aux différentes nations que de diversifier leurs sources d'énergie. L'énergie solaire est une alternative intéressante pour les pays en développement, particulièrement pour les pays subsahariens comme le Burkina Faso à cause de leur important gisement solaire. Avec un rayonnement solaire global moyen de $5,5 \mathrm{kWh} / \mathrm{m} /$ jour et près de $3000 \mathrm{~h}$ d'ensoleillement par an, le Burkina Faso est classé parmi les 25 pays au monde qui tirerait d'énormes profits à l'utilisation des cuiseurs solaires (Solar Cooker International, 2010).
Cependant, les cuiseurs solaires sont peu utilisés au Burkina Faso à cause de plusieurs contraintes dont le manque de données sur l'impact des cuiseurs solaires au Burkina Faso, l'éblouissement lié à leur utilisation, leur encombrement, leur coût élevé, le stockage difficile.

Le cuiseur solaire de type boîte semble être adapté au contexte socio-économique du Burkina Faso dans la mesure où il est facile à construire et à utiliser. Aussi, il peut être conçu majoritairement à base de matériaux locaux et accessibles.

L'objectif de ce travail est de modéliser et simuler le fonctionnement d'un cuiseur solaire de type boîte intégrant des surfaces réflectrices internes et un isolant local sous les conditions météorologiques du Burkina Faso.

Dans un premier temps, l'état des lieux des cuiseurs solaires rencontrés au Burkina Faso ainsi que les contraintes liées à leur utilisation sont présentées. Ensuite, la modélisation des performances d'un cuiseur solaire de type boîte sous les conditions météorologiques du Burkina Faso est présentée. La dernière partie porte sur l'analyse de sensibilité de certains paramètres pour la réalisation du cuiseur. 


\section{ETAT DE L'ART DES CUISEURS SOLAIRES AU BURKINA FASO}

Il existe de nombreux types de cuiseurs. Selon Saxena et al., 2011 les cuiseurs solaires peuvent être classés en quatre types à savoir : les cuiseurs solaires de type boîte, les cuiseurs solaires à concentration, les fours solaires et les cuiseurs solaires indirects.

Les cuiseurs solaires rencontrés ou développés au Burkina Faso sont de plusieurs types. On distingue entre autres: (i) le réflecteur Scheffler (parabolique) (Otte, 2013; Malo, 2015), (ii) le concentrateur hémisphérique (Ky, 2015), (iii) les cuiseurs solaires paraboliques (Tilegwa) et cylindro-parabolique hybride (Tilegwateliman), (iv) le cuiseur Solario (parabolique) et (v) le cuiseur solaire de type boîte développé par l'Institut de Recherche en Sciences Appliquées et Technologies (IRSAT) du Burkina Faso.

La plupart de ces cuiseurs existants connaissent des contraintes qui limitent le développement de la technologie des cuiseurs solaires au Burkina Faso. Il s'agit entre autres de leur coût, de l'éblouissement provoqué par les concentrateurs qui peut endommager les yeux, l'encombrement de ces systèmes et la difficulté à les orienter, la nécessité d'un technicien pour la réparation d'éventuelles pannes, la difficulté de terminer la cuisson à feu doux, le risque de cuisson inachevée quand le ciel se couvre de nuage pendant la cuisine la sensibilité à des vitesses de vents supérieurs à $10 \mathrm{~km} / \mathrm{h}$ (Chandak et al., 2010).

Le cuiseur de type boîte présente beaucoup d'atouts qui peuvent permettre de faire face à ces contraintes. Les travaux entrepris sur le cuiseur de type boîte à l'IRSAT entrent dans ce cadre. Toutefois, plusieurs défis scientifiques restent à relever, notamment l'optimisation de l'isolation, de la captation du rayonnement et le stockage. Ce travail porte sur les deux premiers aspects. Quant à la partie de stockage, elle est en cours de réalisation.

\section{CUISEUR SOLAIRE DE TYPE BOÎTE}

Le cuiseur solaire connu sous le nom de cuiseur solaire boute (fig.1) est de conception simple et facile à construire. Il se compose, généralement, d'une enceinte cubique ou parallélépipédique dont les faces internes sont peintes en noir et isolées thermiquement de tous les côtés. Son fonctionnement est basé sur le principe de l'effet de serre. Il existe aussi les cuiseurs solaires de type boîte à géométrie pyramidale tronquée (Mohammed et al,. 2013, Kumar et al., 2010). Selon Rathore and Warkhedkar, 1995, la durée de cuisson des aliments tels que le riz, les légumes et les légumineuses varie entre $1 \mathrm{~h} 30 \mathrm{~min}$ et $2 \mathrm{~h}$. Le cuiseur solaire de type boîte peut maintenir les aliments à des températures permettant de conserver leur composition biologique pendant une durée de 3h (Funk.and Larson, 1997).

\section{MODÉLISATION DU FONCTIONNEMENT DU CUISEUR SOLAIRE DE TYPE BOÎTE}

\subsection{Description du système}

La figure 1 présente le type de cuiseur qui fait l'objet de ce travail. Il est constitué d'une boîte parallélépipédique à surface d'ouverture inclinée et constituée d'un double vitrage. Il est également muni d'un réflecteur externe qui augmente la quantité d'énergie collectée. Des réflecteurs en aluminium sont collés sur chaque face interne du cuiseur.

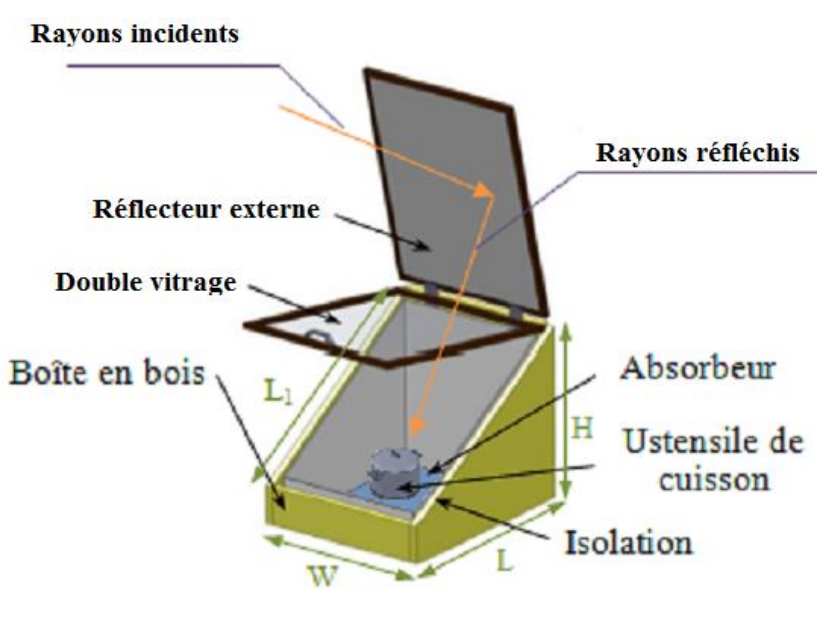

Fig.11. Schéma du dispositif

\subsection{Hypothèses simplificatrices}

Les différents composants du cuiseur solaire sont à des températures uniformes. Les transferts de chaleur convectifs entre les faces internes des parois latérales $\mathrm{du}$ cuiseur et l'air contenu dans le cuiseur sont négligés. Il n’y a pas de gradient de température dans l'épaisseur des vitres. L'échange radiatif entre la plaque absorbante et les parois verticales est négligé. Les propriétés thermo-physiques des matériaux du cuiseur solaire sont constantes dans les plages de température de fonctionnement.

A l'état initial, on a:

$T_{v_{1}}\left(t_{0}\right)=T_{v_{2}}\left(t_{0}\right)=T_{t}\left(t_{0}\right)=T_{a}\left(t_{0}\right)$

$T_{r}\left(t_{0}\right)=T_{f}\left(t_{0}\right)=T_{a b}\left(t_{0}\right)=T_{a m b}\left(t_{0}\right)$

\subsection{Bilan thermique au niveau de chaque composant du cuiseur solaire}

\section{a) Bilan thermique de la vitre externe}

$$
\begin{aligned}
m_{v} c_{v} \frac{\partial T_{v_{1}}}{\partial t}=A_{v} \alpha_{v} G & +h r_{v_{2}-v_{1}} A_{v}\left(T_{v_{2}}-T_{v_{1}}\right)+A_{v} h c_{v_{2}-v_{1}}\left(T_{v_{2}}-T_{v_{1}}\right) \\
& -h r_{v_{1}-c} A_{v}\left(T_{v_{1}}-T_{c}\right)-A_{v} h c_{v_{1}-a m b}\left(T_{v 1}-T_{a m b}\right)
\end{aligned}
$$

$$
m_{v}, c_{v}, A_{v}, \alpha_{v} \text {, désignent respectivement la }
$$

masse, la capacité calorifique, la surface et l'absorptivité de la vitre 
$T_{v_{1}}, T_{v_{2}}, T_{c}, T_{a m b}$, représentent respectivement la température de la vitre externe, de la vitre interne, de la voûte céleste et de l'ambiance.

$h r_{v_{2-} v_{1}}, h r_{v_{1} \_c}$, désignent respectivement les coefficients d'échange thermique par rayonnement entre les deux vitres et entre la vitre externe et la voûte céleste.

$$
h c_{v_{2-} v_{1}}, h c_{v_{1} \_a m b} \text {, représentent respectivement les }
$$
coefficients de transfert thermique par convection entre la lame d'air confinée (entre les deux vitres) et les deux vitres (vitre interne et externe) et entre la vitre externe et le milieu ambiant.

$h c_{v 1 a m b}=\left\{\begin{array}{l}5,621+\frac{1151,2 v}{T_{a m b}} \quad \text { si } v<4,88 m . s^{-1} \\ 604,29\left(\frac{v}{T_{a m b}}\right)^{0,78} \text { si } 4,88<v<30,48 m . s^{-1}\end{array}\right.$

$G$, désigne l'énergie collectée par l'absorbeur :

$$
\begin{aligned}
G= & I_{b} \frac{\cos \theta_{i}}{\sinh }+\frac{1+\cos \beta}{2} I_{d}+\frac{1-\cos \beta}{2} \rho_{a l b} I_{h} \\
& +\rho_{\text {rex }} F_{\text {rex }-v_{1}} I_{b} \cos \theta_{i r}
\end{aligned}
$$

$I_{b}, I_{d}, I_{h}$, représente respectivement l'irradiation solaire directe, diffuse et globale.

$$
\theta_{i}, h, \beta, \theta_{i r} \text {, sont respectivement l'angle d'incidence }
$$
des rayons solaires sur la surface réceptrice du cuiseur, la hauteur solaire, l'angle d'inclinaison de la surface réceptrice du cuiseur et l'angle d'incidence des rayons solaires sur le réflecteur externe.

$\rho_{a l b}, \rho_{\text {rex }}, F_{\text {rex }}$, désigne la réflexivité du sol et du réflecteur externe et la fraction d'énergie réfléchie par le réflecteur externe sur la surface réceptrice du cuiseur.

$$
T_{c}=0,0552 T_{a m b}^{1,5}
$$

\section{b) Bilan thermique de la vitre interne}

$$
\begin{aligned}
& m_{v} c_{v} \frac{\partial T_{v_{2}}}{\partial t}= A_{v}(\alpha \tau) G-h r_{v_{2}-v_{1}} A_{v}\left(T_{v_{2}}-T_{v_{1}}\right) \\
&-A_{v} h c_{v_{2}-v_{1}}\left(T_{v_{2}}-T_{v_{1}}\right)+A_{v} h c_{a-v_{2}}\left(T_{a}-T_{v_{2}}\right) \\
&+h r_{t-v_{2}} A_{t}\left(T_{t}-T_{v_{2}}\right)+h r_{a b-v_{2}}\left(A_{a b}-S_{b}\right)\left(T_{a b}-T_{v_{2}}\right) \\
&-h r_{r-v_{2}} S_{L r}\left(T_{v_{2}}-T_{r}\right) \\
& h c_{a-v_{2}}, \text { désigne le coefficient de transfert }
\end{aligned}
$$

thermique par convection entre l'air à l'intérieur du cuiseur et la vitre interne. $h r_{t-v_{2}}, h r_{r-v_{2}}, h r_{a b-v_{2}}$ sont respectivement les coefficients de transfert thermique par rayonnement entre le couvercle du récipient, le récipient, l'absorbeur et la vitre interne.

$A_{a b}$ est la surface de l'absorbeur.

$A_{t}, S_{b}, S_{L r}$ sont respectivement la surface du couvercle, la surface de base, la surface latérale du récipient.

$T_{a}, T_{a b}, T_{r}, T_{t}$ sont respectivement la température de l'air à l'intérieur du cuiseur, la température de l'absorbeur, la température de la marmite et son couvercle

\section{c) Bilan thermique du couvercle de la marmite}

$$
\begin{aligned}
m_{t} c_{t} \frac{\partial T_{t}}{\partial t} & =A_{t} \alpha_{t} \tau_{v}^{2} G-h r_{t-v_{2}} A_{t}\left(T_{t}-T_{v_{2}}\right) \\
& -A_{t} h c_{t-a}\left(T_{t}-T_{a}\right)-A_{t} h c_{t-f}\left(T_{t}-T_{f}\right) \\
& -h r_{t-f} A_{t}\left(T_{t}-T_{f}\right)
\end{aligned}
$$

$m_{t}$ est la masse du couvercle, $c_{t}$ sa capacité calorifique, $\alpha_{t}$ son absorptivité, $h c_{t-f}$ est le coefficient de transfert thermique entre le couvercle et l'eau dans la marmite, $h r_{t-f}$ est le coefficient de transfert thermique par rayonnement entre le couvercle et l'eau, $T_{f}$ est la température de l'eau.

\section{d) Bilan thermique de la marmite}

$$
\begin{aligned}
m_{r} c_{r} & \frac{\partial T_{r}}{\partial t}=S_{L r} h c_{r-a}\left(T_{a}-T_{r}\right) \\
& +\rho_{r e f} \alpha_{r} G \tau_{v}^{2} \sum_{i=1}^{4}\left(A_{r e f, i} \cos \left(90-\theta_{r e f, i}\right)\right) \\
& +h r_{r-v_{2}} S_{L r}\left(T_{v_{2}}-T_{r}\right)-h c_{r-f} S_{b}\left(T_{r}-T_{f}\right) \\
& +\frac{k_{a b}}{e_{a b}} S_{b}\left(T_{a b}-T_{r}\right)
\end{aligned}
$$

$m_{r}, c_{r}$ sont la masse et la capacité calorifique de la marmite.

$$
\rho_{r e f}, \alpha_{r}, A_{r e f, i}, \theta_{r e f, i} \text { sont respectivement la }
$$
réflexivité des réflecteurs internes, l'absorptivité du récipient, la surface et l'angle d'inclinaison de chaque réflecteur interne. 
$h c_{r-f}, \quad h c_{r-a}$ sont respectivement les coefficients de transfert thermique par convection entre le récipient et l'eau dans le récipient et entre le récipient et l'air à l'intérieur du cuiseur.

$k_{a b}, e_{a b}$ sont la conductivité et l'épaisseur de l'absorbeur.

e) Bilan thermique de l'eau dans la marmite

$$
\begin{gathered}
m_{f} c_{f} \frac{\partial T_{f}}{\partial t}=A_{t} h c_{t-f}\left(T_{t}-T_{f}\right)+h r_{t-f} A_{t}\left(T_{t}-T_{f}\right)(8) \\
+h c_{r-f} S_{L r}\left(T_{r}-T_{f}\right)
\end{gathered}
$$

$m_{f}, c_{f}$ sont la masse et la capacité calorifique de l'eau dans la marmite.

\section{f) Bilan thermique de l'absorbeur}

$$
\begin{gathered}
m_{a b} c_{a b} \frac{\partial T_{a b}}{\partial t}=-h r_{a b-v_{2}}\left(A_{a b}-S_{b a s e}\right)\left(T_{a b}-T_{v_{2}}\right) \\
\quad-\frac{k_{a b}}{e_{a b}} S_{\text {base }}\left(T_{a b}-T_{r}\right)+\alpha_{a b} \tau_{v}^{2}\left(A_{a b}-S_{b a s e}\right) G(9) \\
\quad-A_{a b} h c_{a b-a}\left(T_{a b}-T_{a}\right)-U_{b} A_{a b}\left(T_{a b}-T_{a m b}\right) \\
U_{b}=\left(\left(2 e_{b o i s} / k_{b o i s}\right)+\left(1 / h_{w}\right)+\left(e_{i s} / k_{i s}\right)\right) \\
h_{w}=5,7+3,8 v
\end{gathered}
$$

$m_{a b}, c_{a b}$ sont la masse et la capacité calorifique de l'absorbeur.

$k_{\text {bois }}, k_{\text {is }}$ sont respectivement la conductivité thermique du bois utilisé pour les deux boîtes du cuiseur et de l'isolant.

$e_{\text {bois }}, e_{i s}$ sont respectivement l'épaisseur du bois utilisé pour les deux boîtes du cuiseur et de l'isolant.

\section{g) Bilan thermique de l'air à l'intérieur du cuiseur}

$$
\begin{aligned}
m_{a} c_{a} & \frac{\partial T_{a}}{\partial t}=-A_{v_{2}} h c_{a-v_{2}}\left(T_{a}-T_{v_{2}}\right) \\
& +A_{t} h c_{t-a}\left(T_{t}-T_{a}\right)-S_{L r} h c_{r-a}\left(T_{a}-T_{r}\right) \\
& +A_{a b} h c_{a b-a}\left(T_{a b}-T_{a}\right)-U_{L} S_{L}\left(T_{a}-T_{a m b}\right)
\end{aligned}
$$

$$
U_{L}=\left(\left(e_{\text {bois }} / k_{\text {bois }}\right)+\left(1 / h_{w}\right)+\left(e_{i s} / k_{i s}\right)\right)^{-1}
$$

$m_{r}, c_{r}$ sont la masse et la capacité calorifique de l'air dans le cuiseur.

\subsection{Validation du code numérique}

La figure 2 présente l'évolution de la température de l'eau en fonction du temps dans la marmite obtenue pour notre modèle et celui de Yettou, 2015, qui a étudié expérimentalement un cuiseur solaire de type boîte à surface réceptrice inclinée et à surface réceptrice horizontale. Le cuiseur est incliné d'un angle de $32^{\circ}$. L'évolution de la température d'un litre d'eau dans le récipient posé sur l'absorbeur du cuiseur montre que nos résultats sont en bon accord qualitatif et quantitatif avec ceux de Yettou, 2015. En effet, la valeur maximale et la valeur minimale des écarts sont respectivement de $4,34 \%$ et $0,04 \%$ pour l'eau et de

\begin{tabular}{|c|c|}
\hline Réflecteurs & \\
\hline Réflectivité & $\rho_{\text {ref }}=0,92$ \\
\hline $\begin{array}{c}\text { Surface des réflecteurs } \\
\text { internes }\end{array}$ & $\begin{array}{l}A_{r e f_{1}}=A_{r e f_{2}}=0,11 \mathrm{~m}^{2} \\
A_{r e f_{3}}=0,13 \mathrm{~m}^{2} ; A_{r e f_{4}}=0,065 \mathrm{~m}^{2}\end{array}$ \\
\hline \multicolumn{2}{|l|}{ Plaque absorbante } \\
\hline Matériau de l'absorbeur & Tôle peinte en noir \\
\hline Epaisseur de l'absorbeur & $e_{a b}=0,5 \mathrm{~mm}$ \\
\hline $\begin{array}{c}\text { Absorptivité de } \\
\text { l'absorbeur }\end{array}$ & $\alpha_{a b}=0,93$ \\
\hline Surface de l'absorbeur & $A_{a b}=0,33 \mathrm{~m}$ \\
\hline $\begin{array}{c}\text { Capacité calorifique de } \\
\text { l'absorbeur }\end{array}$ & $C_{a b}=765 J \cdot \mathrm{kg}^{-1} \cdot K^{-1}$ \\
\hline Conductivité thermique & $k_{a b}=250 \mathrm{~W} \cdot \mathrm{m}^{-1} \cdot K^{-1}$ \\
\hline \multicolumn{2}{|l|}{ Vitrage } \\
\hline Epaisseur du vitrage & $4 \mathrm{~mm}$ \\
\hline $\begin{array}{c}\text { Transmissivité de la } \\
\text { vitre }\end{array}$ & $\tau_{v}=0,8$ \\
\hline Surface de la vitre & $A_{v}=0,338 \mathrm{~m}$ \\
\hline Isolation & \\
\hline
\end{tabular}
$5,16 \%$ et $0,08 \%$ pour l'absorbeur du cuiseur.

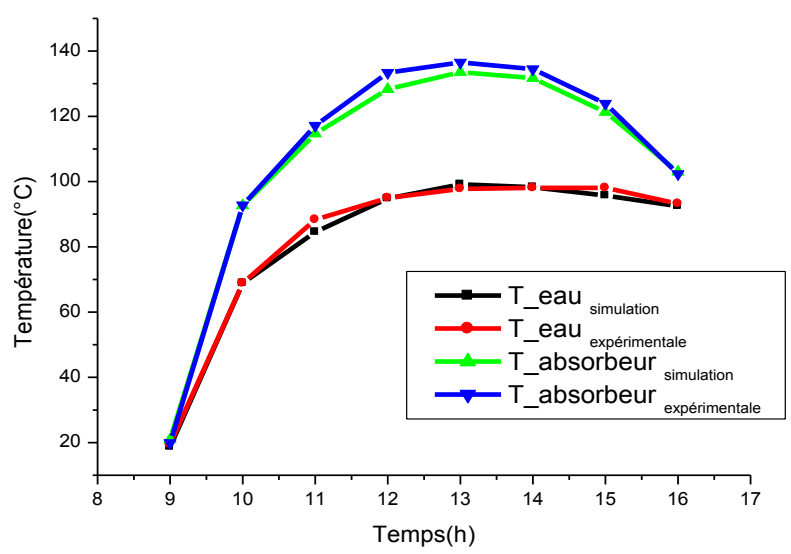

Fig.12. Comparaison des températures de l'eau et l'absorbeur obtenues expérimentalement par Yettou, 2015et la présente simulation.

\section{RÉSULTATS ET DISCUSIONS}

Les données météorologiques utilisées dans ce travail sont celles proposées par Ouedraogo et al., 2012utilisant la notion de jour type.

Tableau1. Paramètres de simulation 


\begin{tabular}{|c|c|}
\hline Matériau d'isolation & Laine de Kapok \\
\hline Conductivité thermique & $k_{i s}=0,035 \mathrm{~W} \cdot \mathrm{m}^{-1} \cdot \mathrm{K}^{-1}$ \\
\hline $\begin{array}{c}\text { Conductivité thermique } \\
\text { du bois }\end{array}$ & $k_{\text {bois }}=0,15 \mathrm{~W} \cdot \mathrm{m}^{-1} \cdot \mathrm{K}^{-1}$ \\
\hline Récipient & $A_{t}=S_{\text {base }}=0,057 \mathrm{~m}^{2}$ \\
\hline Surface de base & $S_{L r}=0,084 \mathrm{~m}^{2}$ \\
\hline Surface latérale & $C_{r}=C_{t}=840 \mathrm{~J} \cdot \mathrm{kg}^{-1} \cdot \mathrm{K}^{-1}$ \\
\hline Capacité calorifique & $m_{r}=0,45 \mathrm{~kg}$ \\
\hline Masse du récipient & $m_{t}=0,15 \mathrm{~kg}$ \\
\hline Masse du couvercle & $\alpha_{r}=\alpha,=0,9$ \\
\hline Absorptivité & \\
\hline Eau & $C_{f}=4185 \mathrm{~J} \cdot \mathrm{kg}^{-1} \cdot \mathrm{K}^{-1}$ \\
\hline Capacité calorifique & $m_{f}=1 \mathrm{~kg}$ \\
\hline Masse &
\end{tabular}

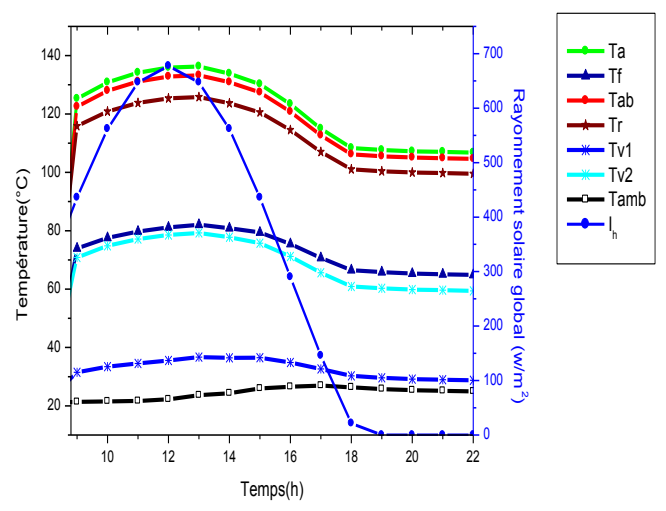

Fig.4a. Evolution de la température des différentes parties du cuiseur pour le mois d'Août

\subsection{Simulation des températures sur toute l'année}

L'évolution temporelle de la température de l'eau dans le cuiseur solaire durant les mois de l'année, est présentée sur les figures 3. Quant aux figures 4a-d, elles présentent l'évolution des températures des différentes parties du cuiseur en fonction du temps et du rayonnement pour le mois d'Août, Avril, Février et Décembre.

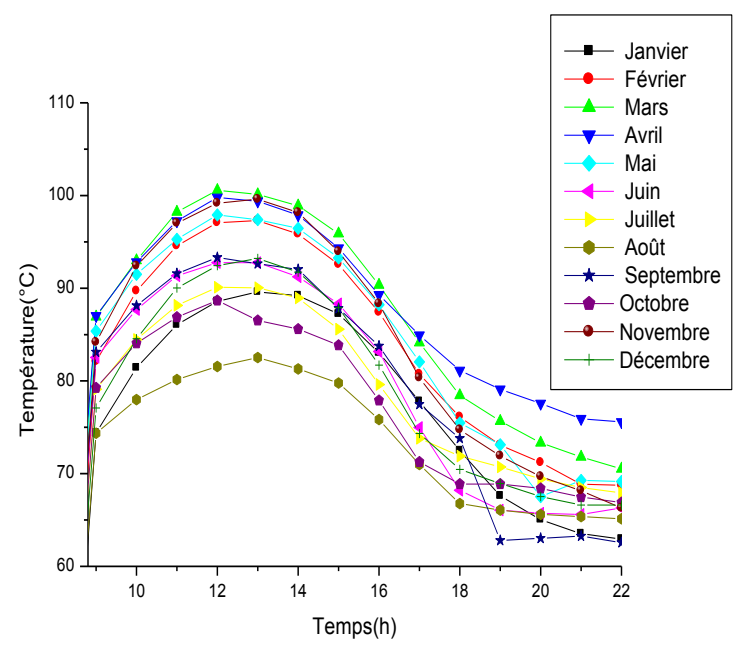

Fig.13. Evolution de la température de l'eau dans le cuiseur le jour type de chaque mois

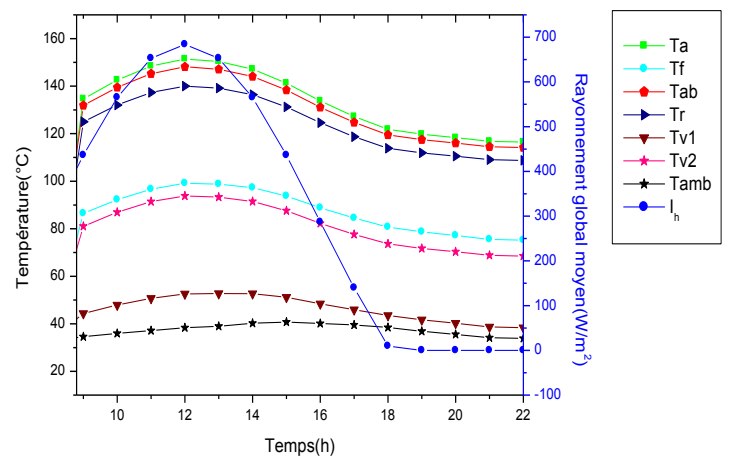

Fig.4b. Evolution de la température des différentes parties du cuiseur pour le mois d'Avril

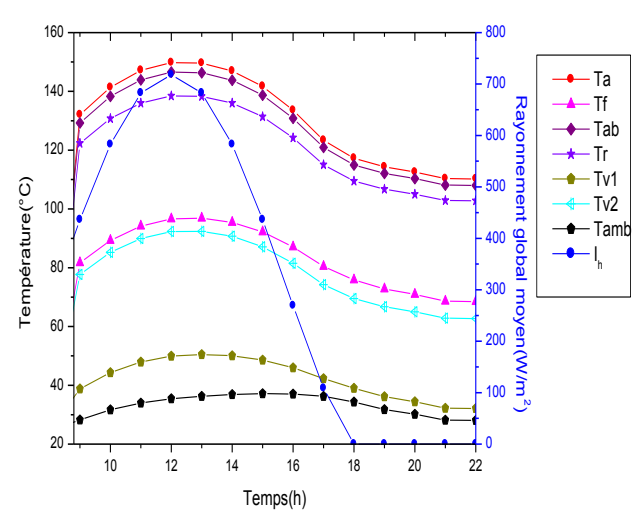

Fig.4c. Evolution de la température des différentes parties du cuiseur pour le mois de Février 


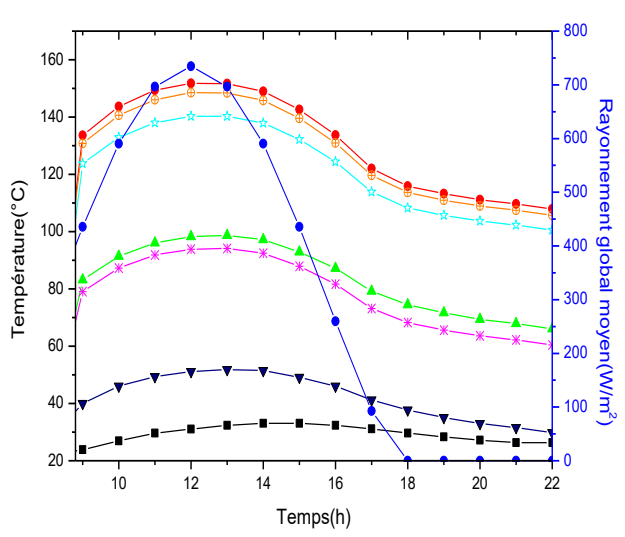

Fig.4d. Evolution de la température des différentes parties du cuiseur pour le mois Décembre

On observe que les courbes ont la même allure. Sous l'action du flux solaire, la température de l'eau croit progressivement jusqu'à atteindre son maximum à $12 \mathrm{~h}$ pour certains mois et à $13 \mathrm{~h}$ pour d'autres. Les mois de la saison sèche présentent les températures les plus élevées. Et elles sont plus importantes pour les mois les plus chauds. Aussi, la température de l'eau de certains mois de la saison pluvieuse (Juin, Juillet et Septembre) avoisine celles de certains mois de la saison sèche (Janvier, Décembre). En Avril, l'eau atteint des températures plus élevées qu'en Décembre quoique le rayonnement solaire est plus important en Décembre qu'en Avril (voir Fig.3 et Fig. 4b et 4d). Tout cela signifie que les performances du cuiseur solaire de type boîte sont dépendantes aussi bien du rayonnement solaire que de la température ambiante. En effet, ce type de cuiseur solaire fonctionne à base de l'effet de serre donc plus l'air ambiant et le rayonnement solaire sont importants, le cuiseur présentera de bonnes performances (Fig. 4a-d). Aussi, ce cuiseur solaire peut fonctionner sous de faibles températures ambiantes. Selon Funk and Larson, 1997, quoique ce type de cuiseur s'échauffe lentement, il fonctionne bien sous une irradiation diffuse, passage nuageux temporaire et une faible température ambiante. A l'exception du mois d'Août, la température varie entre $80^{\circ} \mathrm{C}$ et $100^{\circ} \mathrm{C}$, pendant près de $6 \mathrm{~h}$ (10h à 16h). Selon Solar Cooker International, 2004 , la nourriture commence à cuire entre $82^{\circ} \mathrm{C}$ et $91^{\circ} \mathrm{C}$ dans ce type de cuiseur. Par conséquent, le cuiseur solaire pourrait être un dispositif bénéfique pour les pays sahéliens comme le Burkina Faso.

\subsection{Analyse de sensibilité}

Les différentes courbes de températures du système ayant la même allure, seule la température de l'eau a été utilisée pour l'analyse de sensibilité aux différents paramètres.

\section{a) Influence de l'angle d'inclinaison sur la température de l'eau.}

La figure 5 présente la température de l'eau en fonction du temps pour différents angles d'inclinaison de la surface réceptrice du cuiseur solaire.

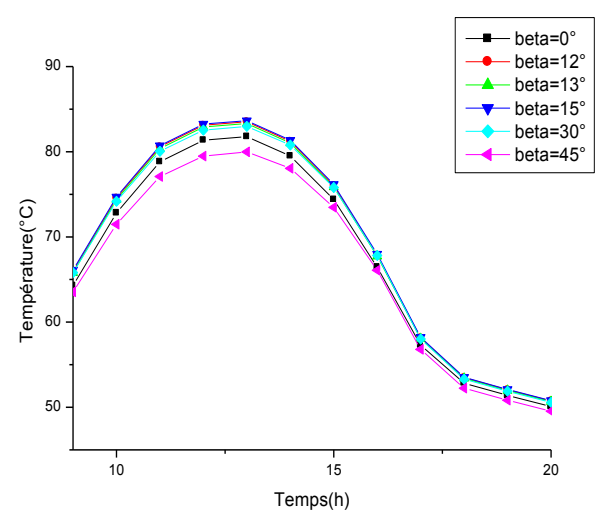

Fig.14. Evolution de la température de l'eau pour différent sangles d'inclinaison de la surface réceptrice du cuiseur

Cette figure indique que pour les angles d'inclinaison proches de la latitude $\left(12,35^{\circ}\right)$, le système atteint les températures les plus élevées. Ce qui confirme la nécessité d'incliner les systèmes de captation de l'énergie solaire d'un angle avoisinant la latitude du lieu. De plus, La figure montre que pour des angles d'inclinaison proche de la latitude du lieu, le cuiseur présente de bonnes performances qu'un cuiseur à surface réceptrice horizontale $\left(\right.$ beta $\left.=0^{\circ}\right)$; ce qui corrobore les résultats obtenus par Yettou, 2015 et Osei and Aidoo, 2018.

\section{b) Influence de l'isolant sur la température de l'eau.}

La figure 6 montre l'impact de l'épaisseur de l'isolant sur les performances du cuiseur solaire.

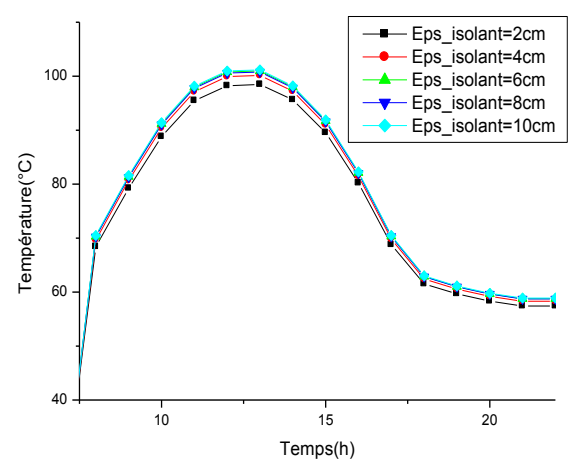

Fig.15.Evolution de la température de l'eau pour différentes épaisseurs de l'isolant

Plus, l'épaisseur de l'isolant est importante, plus le cuiseur atteint, naturellement, des températures importantes. Cela est dû au fait que lorsque l'épaisseur de l'isolant devient grande, le coefficient de 
transmission de la chaleur devient faible. Cependant, à partir de $6 \mathrm{~cm}$, les performances du cuiseur restent presqu'identiques. Ainsi, une épaisseur de $6 \mathrm{~cm}$ de laine de kapok est suffisante pour minimiser les déperditions thermiques dans le cuiseur de type boîte. Au-delà de cette épaisseur, l'amélioration des performances est négligeable. Jubran and Alsaad, 1991obtiennent des résultats similaires pour un cuiseur solaire de type boîte à surface réceptrice horizontale. Thulasi et al., 1994 trouvent qu'une épaisseur de 7,5 $\mathrm{cm}$ d'isolants comme la laine de verre, le papier, la paille, le foin, etc. est adéquate pour les cuiseurs de type boîte à surface horizontale.

\section{c) Influence de la vitesse du vent sur la température de l'eau.}

L'impact de la vitesse du vent sur le cuiseur de type boîte est présenté par la figure 7 .

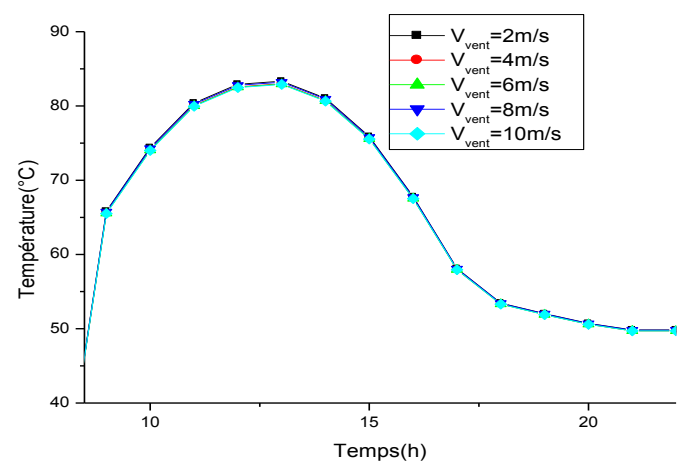

Fig. 7. Evolution de la température de l'eau pour différentes vitesses $d u$ vent

Les résultats montrent que pour des vitesses du vent allant jusqu'à $10 \mathrm{~m} / \mathrm{s}$, le vent n'a aucun impact majeur sur le cuiseur de type boîte. Cela s'explique par le fait que ce système étant isolé de tous les côtés, le vent quel que soit son intensité, ne peut être en contact avec l'air à l'intérieur du cuiseur pour le refroidir. En effet, l'air à l'intérieur du cuiseur joue un rôle important dans l'échauffement de la marmite, puisqu'elle baigne dans cet air. En revanche les cuiseurs solaires de type parabolique sont sensibles à des vitesses de vent supérieures à $10 \mathrm{~km} / \mathrm{h}$ (soit 2,7 m/s) (Chandak et al., 2010). D’une façon générale, la vitesse moyenne mensuelle du vent dans la plupart des villes du Burkina Faso ne dépasse pas $3 \mathrm{~m} / \mathrm{s}$ (données de la météo nationale de 1983 à 1992).

\section{d) Influence de l'épaisseur d'air sur la température de l'eau.}

La figure 8 donne l'évolution temporelle de la température de l'eau, qui est la charge, dans le cuiseur pour différentes épaisseurs de couche d'air entre les deux vitres constituant la surface de captation du cuiseur.

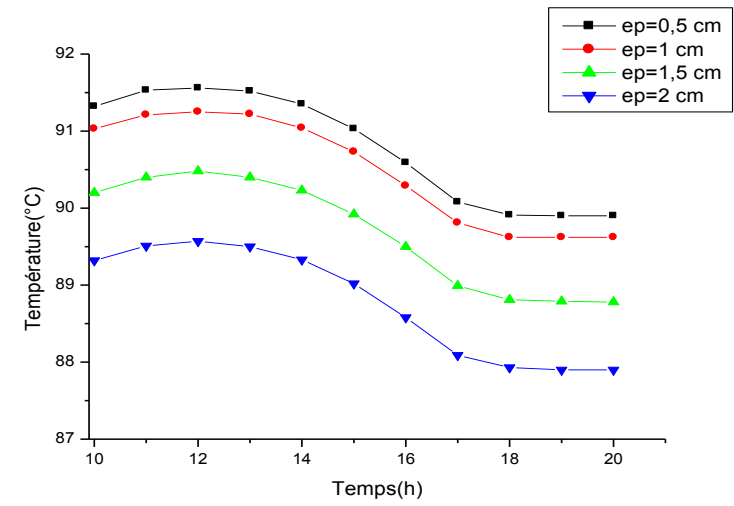

Fig.8. Evolution de la température de l'eau pour différentes épaisseurs de la couche d'air entre les deux vitres

Cette figure montre que la température la plus élevée de l'eau est obtenue pour des épaisseurs de couches d'air relativement faibles. Les résultats indiquent qu'une épaisseur de cette couche d'air comprise entre $0,5 \mathrm{~cm}$ et $1,5 \mathrm{~cm}$ serait appropriée pour le double vitrage de ce type de cuiseur. En effet, l'air est un bon isolant thermique, d'où l'importance de l'utilisation d'un double vitrage pour ce type de cuiseur. Cependant, une épaisseur de la couche d'air inadéquate entre les deux vitres peut engendrer des déperditions thermiques importantes. Si cette épaisseur est inférieure à $0,5 \mathrm{~cm}$, il peut avoir une conduction entre les vitres et si cette épaisseur est aussi grande, la convection entre les vitres peut être très importante, augmentant ainsi les déperditions thermiques. Empiriquement, une épaisseur d'air comprise entre 1 $\mathrm{cm}$ et $2 \mathrm{~cm}$ est proposée par certains auteurs (Saxena et al., 2011).

\section{CONCLUSION}

Ce travail a permis de simuler l'impact des paramètres influents du cuiseur solaire de type boîte sous les conditions météorologiques du Burkina Faso. Ce qui nous a permis de montrer que les performances de ce type de cuiseur dépendent aussi bien de l'intensité du rayonnement solaire que de la température ambiante. Les résultats mettent en évidence que ce cuiseur peut atteindre des températures favorables pour la cuisson solaire pendant toutes les saisons de l'année à Ouagadougou au Burkina Faso. Cependant, le vent n'a pas d'influence majeure sur son fonctionnement. Une épaisseur de la couche d'air entre les deux vitres de la surface réceptrice et une épaisseur d'isolant adéquate peuvent accroitre les performances du cuiseur d'une part et d'autre part minimiser le coût de réalisation du cuiseur.

\section{REMERCIEMENTS:}

Je témoigne toute ma reconnaissance au service de coopération et d'action culturelle (SCAC) de l'ambassade de France au Burkina, pour la bourse 
d'étude qui m'a été octroyée pour effectuer une partie de mes travaux à l'Université de Perpignan via Domitia.

\section{REFERENCES}

Chandak, A., Sardeshpande, V., Keoti, I., Paraboloidal Solar Concentrator, Indian Patent application no. 326/MUM/2010).

Saxena, A., Varun, S.P. Pandey, G. Srivastav, 2011. A thermodynamic review on solar box type cookers, Renewable and Sustainable Energy Reviews 15 3301-3318.

Malo, A.-T., 2015. Les applications de l'énergie solaire thermique dans la cuisson et stockage de chaleur, mémoire de master II professionnel en technologie solaire et appliquée. Université Joseph KI-ZERBO.

Ouedraogo, E., Coulibaly, O., Ouedraogo, A., 2012. Elaboration d'une année météorologique type de la ville de Ouagadougou pour l'étude des performances énergétiques des bâtiments. Revue des Energies Renouvelables, 15, 77-90.

Osei, E.-Y., Amo-Aidoo, A., 2018. Experimental water heating studies of flat box and inclined box solar cooker designs in Ghana; International Journal of Scientific \& Technology Research volume 7, issue 4 ,

Yettou, F., 2015. Conception et réalisation d'un système de cuisson solaire destiné au site saharien (Ghardaïa, Algérie), Thèse de doctorat unique ; Université HADJ LAKHDAR Batna, Algérie.

FAO, 2010. «Foresterie urbaine et périurbaine en Afrique. Quelles perspectives pour le bois-énergie ?» Document de travail sur la foresterie urbaine et périurbaine $\mathrm{n}^{\circ} 4.95$ pages. Rome.
Mohammed, I.-L., Rumah U.-J., Abdulrahim A.-T.; 2013. Performance Testing of a Truncated Pyramid Solar Thermal Cooker; International Journal of Engineering Research and Applications (IJERA), Vol. 3, Issue 4, pp.1174-1178.

Kumar, N., Chavda, T. NMistry H., 2010. A truncated pyramid non-tracking type multipurpose domestic solar cooker/hot water system. Appl Energy; $87: 471-7$.

Rathore, M.-M., Ravi, Dr; Warkhedkar, M., 2015. A review of solar cookers, International Journal of Modern Trends in Engineering and Research, eISSN No.:2349-9745.

Funk, P.-A., Larson, D.-L., 1997. Parametric model of solar cooker performance, Solar Energy 62 (1): $63-$ 8

Pia Piroschka Otte, 2013. Solar cookers in developing countries-What is their key to success, Energy Policy, 63, 375-381.

Solar Cooker International (2010), 25 countries with the greatest potential benefits from solar cookers, disponible en ligne, 2018.

Solar Cooker International, 2004. Cuiseurs solaires : comment construire, employer et apprécier, la $10^{\text {ème }}$ édition.

Thulasi, T.-C., Karmakar, S., Rao, D.-P., 1994. Solar box-cooker: PART II-Analysis and simulation, Solar Energy, Vol. 52, No. 3, pp. 273-282.

Ky T.-S.-M., 2016. Analyse des images optiques des concentrateurs solaires: Application pour la conception de fours à pain, Thèse de Doctorat unique, Université Joseph KI-ZERBO, Burkina Faso 